\title{
ATIVIDADE BIOLÓGICA DO LAPACHOL E DE ALGUNS DERIVADOS SOBRE O DESENVOLVIMENTO FÚNGICO E EM GERMINAÇÃO DE SEMENTES
}

\author{
Marco Andre Alves de Souza*, Andrea Rosane da Silva, Marcio Alcântara Ferreira, Marcela Jacques de Lemos, Rafael \\ Gonçalves Ramos, Aurélio Baird Buarque Ferreira e Sonia Regina de Souza \\ Departamento de Química, Instituto de Ciências Exatas, Universidade Federal Rural do Rio de Janeiro, BR 465, km 7, 23890-000 \\ Seropédica - RJ, Brasil
}

Recebido em 19/9/07; aceito em 14/5/08; publicado na web em 19/9/08

\begin{abstract}
BIOLOGICAL ACTIVITY OF LAPACHOL AND SOME DERIVATIVES AS ANTIFUNGAL AND IN SEED GERMINATION. The natural quinones lapachol, $\alpha$-lapachone and $\beta$-lapachone, and the synthetic derivative $\beta$-lapachone-3-sulfonic-acid were assayed for inhibition of fungal growth (Fusarium oxysporum) and germination of lettuce seeds (Lactuca sativa L.). $\beta$-Lapachone has the strongest activity as a germination inhibitor and lapachol shows no effect. $\beta$-Lapachone, followed by lapachol, are the most active in reducing fungal growth.
\end{abstract}

Keywords: lapachol; Fusarium oxysporum; Lactuca sativa L..

\section{INTRODUÇÃO}

O uso de produtos naturais obtidos de plantas tem se estendido da medicina para a agricultura, onde já foram utilizados para controle de insetos e nematóides, ${ }^{1}$ fungos, ${ }^{2}$ bactérias, ${ }^{3}$ para estimular a imunidade de plantas $^{4}$ e como agentes antigerminativos. ${ }^{5}$

As quinonas representam uma ampla e variada família de metabólitos de distribuição natural. Dentre as naftoquinonas naturais destaca-se o lapachol [2-hidroxi-3-(3-metil-but-2-en-1-il)-1,4naftoquinona, 1], encontrado em árvores do gênero das tabebuias. ${ }^{6}$ A $\beta$-lapachona (2,2-dimetil-3,4-diidro- $2 H$-benzo[ $h]$ cromeno-5,6diona, 2) ${ }^{7}$ e a $\alpha$-lapachona (2,2-dimetil-3,4-diidro- $2 H$-benzo $[g]$ cromeno-5,10-diona, 3) ${ }^{7}$ (Figura 1) também são encontradas no lenho de ipês (Tabebuia), porém em quantidades muito pequenas. Já o ácido $\beta$-lapachona-3-sulfônico (ácido 2,2-dimetil-5,6-dioxo3,4,5,6-tetraidro-2H-benzo[ $h]$ cromeno-3-sulfônico, 4) não é um produto natural. ${ }^{8}$

No âmbito da farmacologia, o lapachol possui ampla atividade biológica contra diferentes organismos. ${ }^{6}$ Recentemente este produto natural vem sendo estudado sob a ótica do combate a fitopatógenos e pragas, em sua ação herbicida e efeitos alelopáticos. ${ }^{9}$

Neste trabalho foram avaliados os efeitos do lapachol e seus derivados sobre o desenvolvimento in vitro do Fusarium oxysporum, um fungo de solo de grande importância agronômica, principalmente por ser sistêmico e de difícil controle químico. Foram também realizados ensaios para avaliar a atividade dessas substâncias sobre a germinação de sementes de alface (Lactuca sativa L.), com o objetivo de se verificar possíveis efeitos de interesse agronômico, como ação estimuladora ou supressora da germinação.

\section{PARTE EXPERIMENTAL}

O lapachol (1) está disponível comercialmente (Aldrich) e foi obtido de madeira de ipê comercial. ${ }^{10}$. As substâncias 2-4 foram preparadas por técnicas descritas ${ }^{7,8}$ (Figura 1). Todos os compostos foram caracterizados espectroscopicamente e os resultados concordam com a literatura. ${ }^{11}$

\footnotetext{
*e-mail: decoerej@yahoo.com.br
}

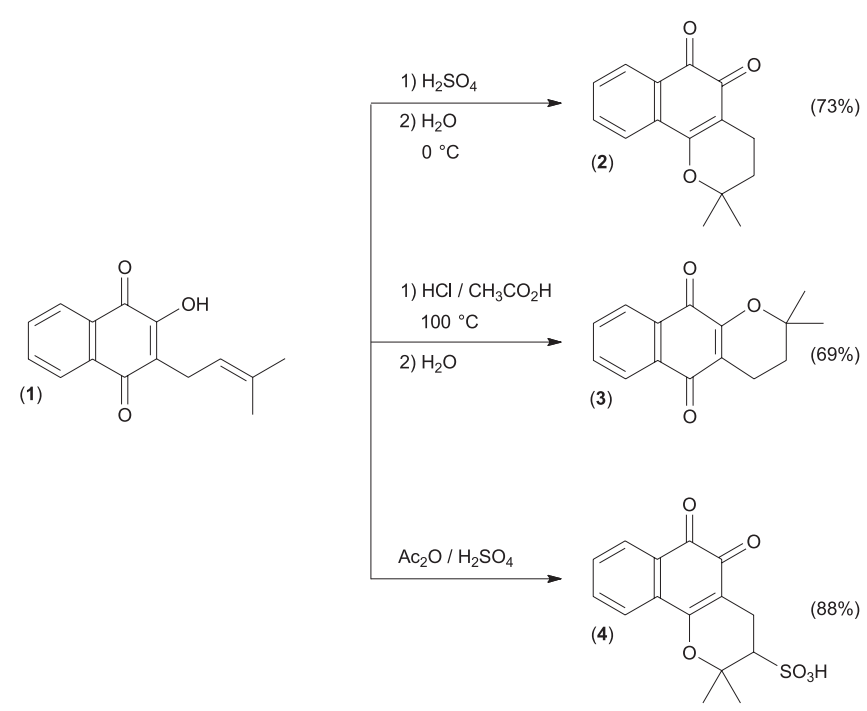

Figura 1. Preparação e estruturas dos compostos formados a partir do lapachol (1): $\beta$-lapachona (2) $\alpha$-lapachona (3) e ácido $\beta$-lapachona-3sulfônico (4)

\section{Material biológico}

Foram utilizadas placas de Petri contendo o fungo Fusarium oxysporum, adquiridas na micoteca da Universidade Federal de Viçosa, identificado com o código de registro DOA-06 e sementes de alface comercial (Lactuca sativa L.).

\section{Preparo das soluções}

\section{Ensaios biológicos}

O ensaio com germinação de sementes de alface empregou soluções de concentração $1 \mathrm{mg} \mathrm{mL}^{-1}$ (em diclorometano (DCM) para 1-3, e em água destilada para 4; os solventes foram escolhidos para proporcionar boa solubilidade). Foram feitos 4 tratamentos, cada um com $2 \mathrm{~mL}$ de cada solução contendo os compostos 1-4, além de 2 controles, um positivo (sem adição) e outro negativo (adição de 2 
$\mathrm{mL}$ de DCM puro). As sementes tratadas foram deixadas ao ar livre para que o solvente evaporasse e, em seguida, adicionaram-se $3 \mathrm{~mL}$ de água destilada para cada placa (exceto o tratamento com $\mathbf{4}$, que recebeu mais $1 \mathrm{~mL}$ de água). Foram feitas 4 repetições para cada tratamento e controle, e cada repetição foi realizada em uma placa de Petri contendo 50 sementes de alface. As placas foram lacradas com filme plástico e acondicionadas em câmara com fotoperíodo de $12 \mathrm{~h}$ (luz do dia) e temperatura de $23 \pm 1^{\circ} \mathrm{C}$.

O ensaio para avaliar o crescimento do fungo $F$. oxysporum empregou soluções a $0,5 \mathrm{mg} \mathrm{mL}^{-1}$ (em dimetilsulfóxido (DMSO) para 1-3, e no próprio meio de cultura para 4), adicionadas na proporção de $1 \%(\mathrm{v} / \mathrm{v})$ ao meio de cultura (batata-dextrose-agar - BDA) para os 4 tratamentos (conc. final no meio de cultura $=5 \mathrm{mg} \mathrm{L}^{-1}$ ); foram feitos 2 controles, um positivo (sem adição) e outro negativo (DMSO puro). Foram feitas 5 repetições para cada tratamento e controle. Em todos os casos foi adicionado o antibiótico de largo espectro Gentamicina ${ }^{\circledR}$. A diluição dos tratamentos no meio de cultura foi realizada alguns instantes antes de usá-los nas placas de Petri. Após verter o meio de cultura nas placas de Petri, fragmentos com estruturas propagativas do fungo (diâmetro = $6 \mathrm{~mm}$ ) foram repicados para as mesmas em capela de fluxo laminar. As placas foram lacradas com filme plástico e acondicionadas em câmara com fotoperíodo de $12 \mathrm{~h}$ (luz do dia) e temperatura de $23 \pm 1{ }^{\circ} \mathrm{C}$.

\section{Coleta de dados e análise}

A germinação foi avaliada no $7^{\circ}$ dia após o início do ensaio. Somente as sementes que apresentaram a radícula e as primeiras duas folhas com padrão normal de crescimento foram aceitas como germinadas.

O desenvolvimento dos fungos foi avaliado diariamente até o $7^{\circ}$ dia, quando as placas do controle positivo estavam com $3 / 4$ da sua área coberta. A medição foi realizada através da média do halo de crescimento, em cm, em dois sentidos ortogonais.

Os dados foram submetidos à análise de variância e as médias comparadas pelo teste de Fisher LSD 5\%.

\section{RESULTADOS E DISCUSSÃO}

\section{Ensaio com germinação}

Não foram observadas variações significativas na germinação das sementes dos controles positivo e negativo (Tabela 1), mostrando que o diclorometano não influenciou na germinação. Entre os tratamentos, o que apresentou maior percentual de inibição da germinação foi o com 2, seguido por 3 e 4; em contrapartida, o tratamento com 1 não apresentou efeito sobre a germinação (Tabela 1).

Quanto à formação das plântulas, os controles e o tratamento com 1 apresentaram plântulas sadias, neste último caso, um pouco menos desenvolvidas; os tratamentos com $\mathbf{4}$ e $\mathbf{2}$ apresentaram plântulas com manchas necróticas no meristema radicular e o tratamento com 3 apresentou plântulas com baixo desenvolvimento do sistema radicular (Figura $2 \mathrm{a}$ ).

A utilização das sementes de alface como parâmetro para avaliar o efeito de substâncias e de extratos de vegetais na germinação é promissor, principalmente em relação a fatores como tempo, custo e espaço. As sementes de alface germinam em poucos dias (em condições ideais, entre 4 e 6 dias), proporcionando rápidos resultados. Estas sementes comerciais podem ser adquiridas com facilidade a custo baixo e os testes podem ser realizados em placas de Petri, facilmente manipuladas na câmara de germinação (Figura 2a).

\section{Ensaio com crescimento fúngico}

Quando houve crescimento do halo fúngico (inclusive no con-
Tabela 1. Número de sementes de alface germinadas e \% de inibição após sete dias

\begin{tabular}{lcccc}
\hline \multicolumn{3}{c}{ Composição da Solução Teste } & $\mathrm{NSG}^{1}$ & $\begin{array}{c}\text { Inibição } \\
(\%)^{2}\end{array}$ \\
\hline Substâncias & $\begin{array}{c}\mathrm{CH}_{2} \mathrm{Cl}_{2} \\
(\mathrm{~mL})\end{array}$ & $\begin{array}{c}\text { Concentração } \\
\left(\mathrm{mg} \mathrm{L}^{-1}\right)\end{array}$ & & \\
\hline$(\mathbf{C}+)$ & - & - & $49,0 \mathrm{a}$ & - \\
$(\mathbf{C}-)$ & 2 & - & $47,3 \mathrm{a}$ & 3,6 \\
$(\mathbf{1})$ & 2 & 1000 & $44,5 \mathrm{a}$ & 9,2 \\
$(\mathbf{2})$ & 2 & 1000 & $24,8 \mathrm{c}$ & 49,5 \\
$(3)$ & 2 & 1000 & $31,5 \mathrm{~b}$ & 35,7 \\
$(4)$ & - & 1000 & $33,8 \mathrm{~b}$ & 31,1 \\
\hline
\end{tabular}

': número de sementes germinadas (valor médio); letras iguais na mesma coluna indicam diferenças não significativas (Fisher LSD $5 \%){ }^{2}$ : em função do número de sementes germinadas do controle positivo.

$\mathbf{a}$
$(\mathrm{C}+)$

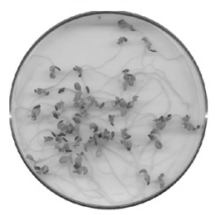

$(\mathrm{C}+)$

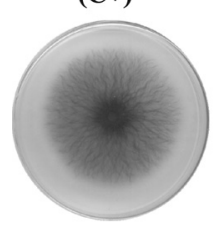

(2)

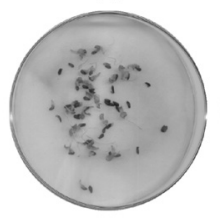

(1)

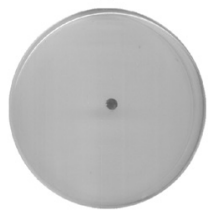

b
(3)
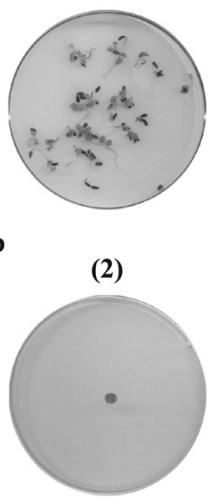

(4)

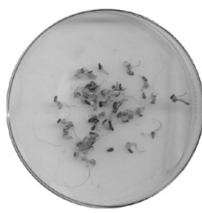

(3)

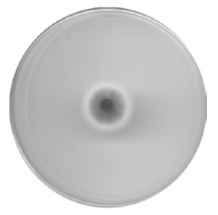

Figura 2. Germinação de alface e crescimento fúngico em placas de Petri. a: imagens (obtidas no $7^{\circ}$ dia) de sementes de alface submetidas a: $\left(C^{+}\right)$: controle positivo; (2): $\beta$-lapachona; (3): $\alpha$-lapachona; (4): ácido $\beta$-lapachona-3sulfônico. Os tratamentos com lapachol (1) e o controle negativo mostraram-se indistinguíveis de $C+$; $b$ : imagens (obtidas no 7 o dia) do desenvolvimento do fungo F. oxysporum submetido a: $(C+)$ : controle positivo; (1): lapachol; (2): $\beta$-lapachona; (3): $\alpha$-lapachona. Os tratamentos com ácido $\beta$-lapachona-3sulfônico (4) e o controle negativo mostraram-se indistinguíveis de C+

trole negativo, apenas com DMSO) não foram observadas alterações morfológicas no desenvolvimento dos fungos.

Não houve diferença significativa entre os controles positivo e negativo no $7^{\circ}$ dia após o início do ensaio (Tabela 2), comprovando que o DMSO utilizado a $1 \%\left(\mathrm{v} \mathrm{v}^{-1}\right)$ no meio de cultura pode ser utilizado como veículo às substâncias insolúveis em meio aquoso.

$\mathrm{O}$ tratamento com 4 não apresentou efeito inibitório, no $7^{\circ}$ dia, se comparado aos controles (Tabela 2). As substâncias 1-3 inibiram o crescimento fúngico e, dentre estas, 2 proporcionou $95,5 \%$ de inibição (Tabela 2).

Os efeitos das substâncias testadas foram meramente fungistáticos, ou seja, não destroem por completo o micélio fúngico. Na Figura $2 \mathrm{~b}$ podem-se observar dois casos em que praticamente não houve crescimento fúngico, entretanto existe um curto micélio formado por hifas hialinas que não são perceptíveis nas imagens.

Os ensaios descritos confirmam resultados preliminares ${ }^{12}$ em que foi relatada a atividade inibitória do lapachol sobre o crescimento dos fungos Fusarium oxysporum, Rhizoctonia solani e Sclerotium rolfsii em placas de Petri. Apesar da vasta literatura citada por Hussain ${ }^{6}$ e Sil$\mathrm{va}^{7}$ quanto aos efeitos farmacológicos do lapachol e derivados, poucos 
Tabela 2. Halo de crescimento e percentual de inibição do crescimento de $F$. oxysporum após sete dias

\begin{tabular}{|c|c|c|c|c|}
\hline \multicolumn{3}{|c|}{ Composição da Solução Teste } & \multirow{2}{*}{$\begin{array}{l}\text { Halo } \\
(\mathrm{cm})^{2}\end{array}$} & \multirow{2}{*}{$\begin{array}{c}\text { Inibição } \\
(\%)^{3}\end{array}$} \\
\hline Tratamento & $\begin{array}{l}\text { DMSO } \\
(\%)\end{array}$ & $\begin{array}{l}\text { Concentração } \\
\left(\mathrm{mg} \mathrm{L}^{-1}\right)^{1}\end{array}$ & & \\
\hline$(\mathrm{C}+)$ & - & - & $6,90 \mathrm{a}$ & - \\
\hline$(\mathrm{C}-)$ & 1 & - & $5,90 \mathrm{a}$ & 14,5 \\
\hline (1) & 1 & 5 & $0,50 \mathrm{~b}$ & 92,8 \\
\hline (2) & 1 & 5 & $0,31 \mathrm{~b}$ & 95,5 \\
\hline (3) & 1 & 5 & $1,48 \mathrm{~b}$ & 78,6 \\
\hline (4) & - & 5 & $5,85 \mathrm{a}$ & 15,2 \\
\hline
\end{tabular}

${ }^{1}$ : concentração final no meio de cultura. ${ }^{2}$ : letras iguais na mesma coluna indicam diferenças não significativas (Fisher LSD 5\%). ${ }^{3}$ : inibição (\%) em função do halo de crescimento do controle positivo.

são, ainda, os trabalhos que buscam um enfoque agronômico. ${ }^{9}$

\section{CONCLUSÃO}

De todas as substâncias testadas o lapachol foi a que apresentou as melhores características para uso agronômico, uma vez que não altera o percentual de germinação de sementes comerciais de alface, mas inibe o crescimento fúngico, evitando o tombamento de mudas (causado pelo fungo F. oxysporum).

\section{MATERIAL SUPLEMENTAR}

Está disponível em http://quimicanova.sbq.org.br, na forma de arquivo PDF, com acesso gratuito.

\section{REFERÊNCIAS}

1. Saito, M. L; Luchini, F.; Substâncias obtidas de plantas e a procura por praguicidas eficientes e seguros ao meio ambiente, $1^{\text {a }}$ ed., EMBRAPACNPMA: Jaguariúna, 1998; Resumos do $1^{o}$ encontro de processos de proteção de plantas: controle ecológico de pragas e doenças, Hein, M., org.; Ed. Agroecológia: Botucatu, 2001.

2. Chalfoun, S. M.;Carvalho, V. D.; Fitopatol. Bras. 1987, 12, 230; Stangarlin, J. R.; Schwan-Estrada, K. R. F.; Cruz, M. E. S.; Nozaki, M. H.; Biotecnologia, Ciência e Desenvolvimento 1999, 11, 16; Souza, M. A. A.; Souza, S. R.; Veiga-Junior, V. F.; Cortez, J. K. P. C.; Leal, R. S.; Dantas, T. N. C.; Maciel, M. A. M.; Rev. Bras. Farmacogn. 2006, 16, 599.

3. Cordeiro, C. H. G.; Sacramento, L. V. S.; Corrêa, M. A.; Pizzolitto; A. C.; Lara, E. H. G.; Moraes, H. P.; Rev. Bras. Pl. Med. 2006, 8, 173.

4. Smith, C. J.; New Phytologist 1996, 132, 1.

5. Pires, N. M.; Prates, H. T.; Pereira Filho, I. A.; Oliveira Jr, R. S.; Faria, T. C. L.; Scientia Agricola 2001, 58, 61; Piña-Rodrigues, F. C. M.; Lopes, B. M.; Floresta e Ambiente 2001, 8, 130; Prates, H. T.; Paes, J. M. V.; Pires, N. M.; Pereira Filho, I. A.; Magalhães, P. C.; Pesq. Agropec. Bras. 2000, 35, 909; Soares, G. L. G.; Vieira, T. R.; Floresta e Ambiente 2000, $7,180$.

6. Hussain, H.; Krohn, K.; Ahmad, V. U.; Miana, G. A.; Green, I. R.; ARKIVOC 2007 (ii), 145.

7. Hooker, S. C.; J. Am. Chem. Soc. 1936, 58, 1190; Silva, M. N.; Ferreira, V. F.; Souza, M. C. B. V.; Quim. Nova 2003, 26, 407.

8. Fieser, L. F.; J. Am. Chem. Soc. 1948, 70, 3232.

9. Spencer, G. F.; Tjarks, L. W.; England, R. E.; Seest, E. P.; J. Nat. Prod. 1986, 49, 530; Ali, R. M.; Houghton, P. J.; Hoo, T. S.; Phytoter. Res. 1998, 12, 331 .

10. Paternò, E.; Gazz. Chim. Ital. 1882, 12, 337.

11. Ferreira, C. A. C.; Ferreira, V. F.; Pinto, A. V.; Lopes, R. S. C.; Pinto, M. C. R.; Silva, A. J. R.; An. Acad. Bras. Ci. 1987, 59, 5; Pinto, A. V.; Pinto, M. C. F. R.; Oliveira C. G. T.; An. Acad. Bras. Ci. 1982, 54, 108; Andrade-Neto, V. F.; Goulart, M. O. F.; Silva Filho, J. F.; Silva, M. J.; Pinto, M. C. F. R.; Pinto, A. V.; Zalis, M. G.; Luzia H. Carvalho, L. H.; Krettli, A. U.; Bioorg. Med. Chem. Lett. 2004, 14, 1145.

12. Araújo, O. J. L.; Ferreira, M. A; Souza, M. A. A.; Stark, E. M. L. M.; Souza, S. R.; Anais da XIV Jornada de Iniciação Científica da UFRRJ, Seropédica, Brasil, 2004. 


\section{ATIVIDADE BIOLÓGICA DO LAPACHOL E DE ALGUNS DERIVADOS SOBRE O DESENVOLVIMENTO FÚNGICO E EM GERMINAÇÃO DE SEMENTES}

Marco Andre Alves de Souza*, Andrea Rosane da Silva, Marcio Alcântara Ferreira, Marcela Jacques de Lemos, Rafael Gonçalves Ramos, Aurélio Baird Buarque Ferreira e Sonia Regina de Souza

Departamento de Química, Instituto de Ciências Exatas, Universidade Federal Rural do Rio de Janeiro, BR 465, km 7, 23890-000 Seropédica - RJ, Brasil

As preparações e os dados espectroscópicos das substâncias 1-4 estão distribuídos na literatura, em alguns casos em teses de difícil acesso. Para maior comodidade, estão reunidas a seguir estas informações. (Os espectros foram feitos nos seguintes equipamentos: ${ }^{1} \mathrm{H}$ e ${ }^{13} \mathrm{C}$ RMN: Bruker Ac 200 ou Bruker Avance 500 ( $\mathrm{CDCl}_{3}$, TMS); I.V.: Perkin-Elmer 1605 (pastilhas de KBr); CG-EM-IE: Varian Saturn 2000 (70 eV, coluna VF5MS) e E.M.-E.S.(electrospray): Micromass ZQ).

Extração do lapachol (1): Os veios amarelos no lenho do ipê são selecionados e moídos. Extrai-se com sol. aq. a 2,5\% de $\mathrm{Na}_{2} \mathrm{CO}_{3}(4$ $\mathrm{L}$ sol./kg de pó). Filtra-se a solução vermelho-vinho e neutraliza-se com $\mathrm{HCl}$ ou $\mathrm{H}_{2} \mathrm{SO}_{4} 1 \mathrm{~N}$ (na neutralização desaparece a cor avermelhada e surgem grumos amarelos). Filtra-se a pasta amarelada de lapachol, que é recristalizado de etanol. (Rendimento $\approx 1,5 \%(\mathrm{p} / \mathrm{p})$.) IV: $3351-2967 ; 2914-2853 ; 1642 ; 1589 ; 791-724 \mathrm{~cm}^{-1} .{ }^{1} \mathrm{H}$ RMN: $\delta$ $8,1$ (dd, $J=1,4 ; 6,2 ; 1 \mathrm{H}) ; 8,0(\mathrm{dd}, J=1,4 ; 7,6 ; 1 \mathrm{H}) ; 7,7$ (dt, $J=1,4$; $6,2 ; 1 \mathrm{H}) ; 7,6(\mathrm{dt}, J=1,4 ; 7,3 ; 1 \mathrm{H}) ; 5,1(\mathrm{t}, J=7,3 ; 1 \mathrm{H}) ; 3,3(\mathrm{~d}, J=$ $7,3 ; 2 \mathrm{H}) ; 1,7$ (s, 3H); 1,6 (s, 3H). ${ }^{13} \mathrm{C}$ RMN: $\delta 185,5 ; 181,6 ; 152,6$; 134,$7 ; 133,8 ; 132,8 ; 129,3 ; 126,7 ; 126,0 ; 119,6 ; 25,7 ; 22,6 ; 17,8$. EM (IE): $m / z$ (\%)242 (15); 228 (16); 227 (100); 199 (7); 115(5); 105(4); 77(7). P.f.: $139-140{ }^{\circ} \mathrm{C}$.

Síntese da $\beta$-lapachona (2): Em um bécher em banho de gelo, adiciona-se $5 \mathrm{~g}$ de lapachol a $15 \mathrm{~mL}$ de ácido sulfúrico concentrado gelado, de uma só vez. Agita-se por aproximadamente 10 min e vertese em $500 \mathrm{~mL}$ de água destilada gelada. Filtra-se o sólido laranja e recristaliza-se de etanol. (Rendimento: 70\%.) IV: 2975-2928; 1639 e 1596; $1565 ; 1224 ; 895 \mathrm{~cm}^{-1} .{ }^{1} \mathrm{H}$ RMN: $\delta 8,0(\mathrm{dd}, J=1,4 ; 6,9 ; 1 \mathrm{H}) ; 7,8$ $(\mathrm{dd}, J=1,1 ; 7,7 ; 1 \mathrm{H}) ; 7,6(\mathrm{dt}, J=1,3 ; 7,4 ; 1 \mathrm{H}) ; 7,5(\mathrm{dt}$, $J=1,1 ; 7,4 ; 1 \mathrm{H}) ; 2,5(\mathrm{t}, J=6,7 ; 2 \mathrm{H}) ; 1,8(\mathrm{t}, J=6,7 ; 2 \mathrm{H})$; $1,4(\mathrm{~s}, 6 \mathrm{H}) .{ }^{13} \mathrm{C}$ RMN : $\delta 179,5 ; 178,4 ; 162,0 ; 134,7 ; 132,5$; 130,$5 ; 130,0 ; 128,4 ; 124,0 ; 112,6 ; 79,2 ; 31,4 ; 26,6 ; 16,0$. EM (IE): $m / z$ (\%) 242 (100); 227 (10); 214 (40); 199 (20) ; 158 (40); 159 (100); 181 (20); p.f.: $154-156{ }^{\circ} \mathrm{C}$.

Síntese da $\alpha$-lapachona (3): Em um balão, dissolvem-se $5 \mathrm{~g}$ de lapachol em uma mistura de $5 \mathrm{~mL}$ de ácido acético glacial e $13 \mathrm{~mL}$ de $\mathrm{HCl}$ conc. Após $1,5 \mathrm{~h}$ de aquecimento a $100{ }^{\circ} \mathrm{C}$ com agitação, adiciona-se água até completar a precipitação do sólido amarelo claro, que é filtrado e recristalizado de etanol. (Rendimento: 68\%.) IV: 2972-2938; 1681 e 1638; 1573; 1268; $885 \mathrm{~cm}^{-1} .{ }^{1} \mathrm{H} \mathrm{RMN}$ : $\delta 8,0(\mathrm{~m}, 2 \mathrm{H}) ; 7,6(\mathrm{~m}, 2 \mathrm{H}) ; 2,5(\mathrm{t}, J=6,7 ; 2 \mathrm{H}) ; 1,8(\mathrm{t}, J=6,7 ; 2 \mathrm{H})$; $1,4(\mathrm{~s}, 6 \mathrm{H}) ;{ }^{13} \mathrm{C}$ RMN: $\delta 184,4 ; 180,0 ; 154,6 ; 133,9 ; 132,9 ; 132,1$; 131,$2 ; 126,0 ; 126,3 ; 120,2 ; 78,2 ; 31,5 ; 26,5 ; 16,8$. EM (IE): $\mathrm{m} / z(\%)$ 242 (40); 227 (100); 209 (5); 199 (10); 181 (10). P.f.: 119-120 ${ }^{\circ} \mathrm{C}$.

Síntese do ácido $\beta$-lapachona-3-sulfônico (4): Agita-se uma suspensão de 2,42 g de lapachol em anidrido acético $(10 \mathrm{~mL})$ e adicionase gota a gota $0,6 \mathrm{~mL}$ de $\mathrm{H}_{2} \mathrm{SO}_{4}$ conc., mantendo-se a temperatura entre 20 e $30{ }^{\circ} \mathrm{C}$. O produto vermelho precipita e a reação termina com o desaparecimento do lapachol na cromatografia em camada fina. Após resfriar, filtra-se e lava-se com éter seco. (Rendimento: $88 \%$.) IV: $3544-3353 ; 1653$ e 1596; 1565; 1240; $281 \mathrm{~cm}^{-1} .{ }^{1} \mathrm{H} \mathrm{RMN}: \delta 7,9$ $(\mathrm{d}, J=7,2 ; 1 \mathrm{H}) ; 7,7(\mathrm{~m}, 2 \mathrm{H}) ; 7,6(\mathrm{t}, J=6,0 ; 1 \mathrm{H}) ; 2,8(\mathrm{~m}, 2 \mathrm{H}) ; 2,4(\mathrm{~m}$, $1 \mathrm{H}) ; 1,7$ (s, 3H) ;1,4 (s, 3H). ${ }^{13} \mathrm{C}$ RMN: $\delta 179,0 ; 177,5 ; 159,7 ; 134,9$; 131,$8 ; 130,7 ; 129,9 ; 127,7 ; 123,7 ; 112,8 ; 81,9 ; 59,4 ; 28,6 ; 21,0 ; 20,4$. EM (ES, íons negativos) $\mathrm{m} / \mathrm{z}(\%) ; 321,2$ ([M-1]; 100).

*e-mail: decoerej@yahoo.com.br 